\title{
Publisher Correction: A diverse range of factors affect the nature of neural representations underlying short-term memory
}

\section{A. Emin Orhan D and Wei Ji Ma}

Correction to: Nature Neuroscience https://doi.org/10.1038/s41593-018-0314-y, published online 21 January 2018.

In the version of this article initially published, errors occurred in Fig. 5: in panel a, the leftmost label on the $x$ axis should have read "COMP $\rightarrow 2 \mathrm{AFC"}$ and the rightmost $x$ axis marks in Fig. $5 \mathrm{c}$ should have been labeled "1.12". The errors have been corrected in the HTML and PDF versions of the article.
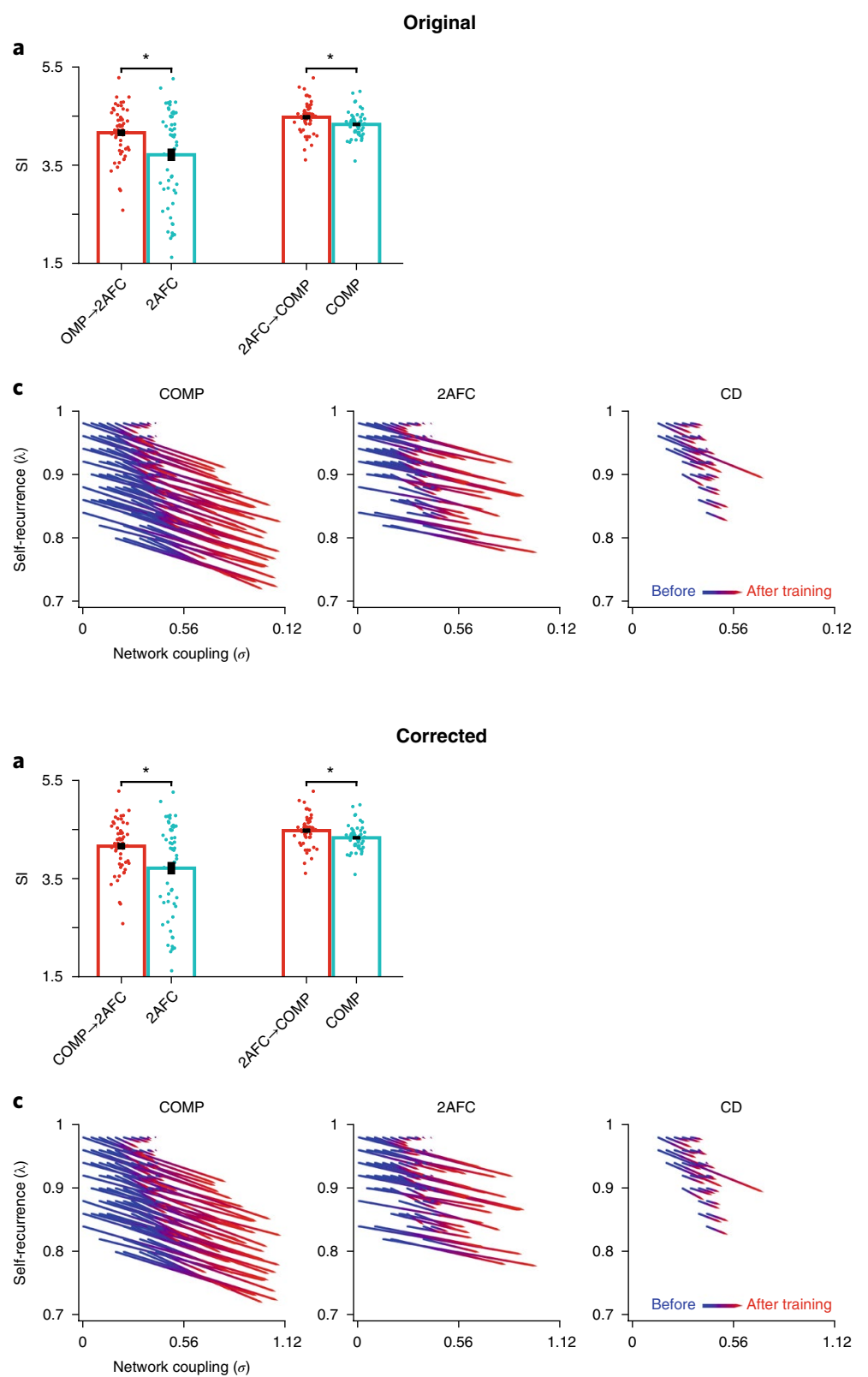

Fig. 5 | Original and Corrected. 